\title{
Síndrome de fragilidade aumenta a suscetibilidade à ventilação mecânica?
}

\section{Fraglity syndrome increases susceptibility to mechanical ventilation?}

\author{
Sandra Lisboa', Bruno Rodrigues Rosa ${ }^{2}$, Christine Pereira Gonçalves ${ }^{3}$, Cid Marques David ${ }^{4}$
}

\begin{abstract}
RESUMO
Alguns marcadores clínicos da síndrome de fragilidade, como desnutrição, sarcopenia e delírio são preditores de insuficiência respiratória em idosos. Dessa forma, esses fatores de risco podem levar os pacientes considerados frágeis a uma maior suscetibilidade à intubação e ventilação mecânica. Objetivo: identificar evidências de associação causal entre a síndrome de fragilidade e ventilação mecânica invasiva. Métodos: busca no MEDLINE por coortes que demonstrem que a síndrome de fragilidade aumenta o risco de intubação e ventilação mecânica invasiva. Além do termo síndrome de fragilidade, foram incorporados e considerados também os marcadores clínicos da síndrome que podem apresentar associação à insuficiência respiratória, reconhecidos na literatura como sarcopenia, delírio e desnutrição. A busca foi realizada através de estratégia de busca específica, incluindo os termos de busca (Medical Subject Headings - MeSH) e respectivos sinônimos. Além disso, foi realizada uma busca adicional na lista de referências dos estudos incluídos. Resultados: nenhum estudo preencheu os critérios de inclusão desta revisão. Conclusões: Em nosso estudo, não foram encontradas evidências de que a síndrome de fragilidade possa ser um fator de risco para ventilação mecânica invasiva. Existem indícios, no entanto, de que o delírio possa aumentar o tempo de um paciente submetido à ventilação mecânica. Apesar disso, há considerável carência de informações nessa área e muita incerteza, talvez por ainda não haver um consenso estabelecido sobre a síndrome. Há uma expressa necessidade de realização de estudos que avaliem se marcadores clínicos da síndrome de fragilidade são fatores de risco para ventilação mecânica invasiva.
\end{abstract}

Palavras-chave: Idoso Fragilizado. Envelhecimento. Cuidados Intensivos. Ventilação Mecânica.

1.Fisioterapeuta; Doutoranda do Curso de Pós-graduação em Clínica Médica - UFRJ; Chefe do Setor de Provas de Função Respiratória do Instituto Fernandes Figueira - IFF/ FIOCRUZ;

2. Fisioterapeuta; Mestrando do Curso de Pós-graduação em Medicina Interna e Terapêutica, e Medicina Baseada em Evidências - UNIFESP; Assistente de pesquisa do Centro Cochrane do Brasil;

3. Doutora em Ciências Fisiológicas; Fisioterapeuta do Instituto Fernandes Figueira/ Fundação Oswaldo Cruz - IFF/ FIOCRUZ;

4. Médico Pneumologista; Doutor em Doenças Infecciosas e Parasitárias, e Professor Associado 2 do Departamento de Clínica Médica da Faculdade de Medicina - UFRJ.
Correspondência:

Sandra Lisboa

Fundação Oswaldo Cruz - Instituto Fernandes Figueira Setor de Provas de Função Respiratória

Avenida Rui Barbosa, 716

22250-020 - Rio de Janeiro, Brasil

Tel.: (+55) 2125541852

Email: sandralisboaft@ terra.com.br

Artigo recebido em 26/04/2010 Aprovado para publicação em 11/06/2010 
Algumas características são fortes marcadores da síndrome de fragilidade, como desnutrição, dependência funcional, repouso prolongado, úlceras de pressão, distúrbios da marcha, fraqueza generalizada, idade $>90$ anos, perda de peso, anorexia, medo de cair, demência, fraturas, sarcopenia, delírio, confusão e respirar ar puro com pouca frequiência. ${ }^{1,2}$ Tais marcadores são resultantes, com o processo de envelhecimento, dos efeitos da falta de exercício físico, alimentação inadequada, ambiente insalubre, lesões, doenças e drogas. Existem, ainda, fortes mecanismos moleculares envolvidos no processo desta síndrome. Existem evidências de que alterações na função mitocondrial em muitos tipos de tecidos foram consideradas como um possível mecanismo causador da síndrome de fragilidade. ${ }^{3}$ Todos esses fatores interligados levam à desnutrição crônica, consolidada por mudanças relacionadas à idade, causando a perda de osso e, principalmente, de massa muscular esquelética, definida como sarcopenia. ${ }^{1}$ Sarcopenia, por sua vez, é o processo pelo qual ocorre a perda degenerativa de massa e força nos músculos com o envelhecimento, resultando em um aumento da sensação de esforço para uma dada intensidade de exercício. ${ }^{4}$ Declínio na função muscular pode causar uma redução na força, incluindo o diafragma e a musculatura respiratória acessória, predispondo o aparecimento da fadiga, quando o trabalho ventilatório é aumentado pela doença. Subsequentemente, esse processo pode comprometer a reserva cardiopulmonar substancialmente e uma infecção ou insulto agudo que aumenta a demanda metabólica pode precipitar uma situação de insuficiência respiratória. Além disso, pacientes com distúrbios neurológicos, como o delírio apresentam risco aumentado para o desenvolvimento de aspiração, que é um freqüente causador de insuficiência respiratória. ${ }^{5}$ Delírio é diagnosticado em cerca de $67 \%$ dos pacientes ventilados mecanicamente. ${ }^{6,7,8}$ Delírio, na UTI, está associado a aumento do tempo sob. ventilação mecânica ${ }^{6,7,9}$ e, dessa forma, tais pacientes ficam mais vulneráveis a complicações da própria ventilação mecânica, como aspiração e pneumonia nosocomial. ${ }^{9}$ Contextualmente, desnutrição, sarcopenia e delírio são marcadores da síndrome de fragilidade ${ }^{10}$ que, associados à idade avançada, são fortes preditores de insuficiência respiratória. ${ }^{5}$ Interação desses fatores pode culminar com a vulnerabilidade fisiológica resultante da prejudicada reserva homeostática e subsequente redução da capacidade do organismo para suportar o estresse. Assim, pessoas frágeis tem risco aumentado para efeitos adversos, como internação hospitalar em longo prazo e cuidados intensivos. ${ }^{10,11,12}$ Sarcopenia, por exemplo, resulta em aumento do risco a quedas e fraturas, podendo levar a internação hospitalar e acompanhamento médico. ${ }^{13}$ Desnutrição e delírio são preditores de insuficiência respiratória, além da própria sarcopenia, o que constitui outra situação clínica potencialmente tratada em ambiente hospitalar. Fried et al. complementam que pacientes fisicamente frágeis são os mais prováveis de serem hospitalizados. Em uma coorte de 3782 idosos considerados frágeis, 931 (25\%) foram hospitalizadas pelo menos uma vez em um período de 6 meses. Através de um modelo de regressão logística, comprometimento funcional grave (odds ratio - 1.20, IC 95\% - 1.01, 1.43), piora do desempenho para atividades da vida diária (odds ratio - 1,22, IC 95\% 1.04, 1.43), presença de um úlcera de decúbito (odds ratio 1,62, IC 95\% 1.17, 2.24), diagnóstico primário de insuficiência cardíaca congestiva (odds ratio - 1,61, IC 95\% $1.11,2.34)$ e diagnóstico primário de doença respiratória (odds ratio - 1,77, IC 95\% 1.24, 2.54) foram associados à hospitalização. ${ }^{14} \mathrm{O}$ objetivo desse estudo é identificar evidências de que a síndrome de fragilidade possa aumentar a probabilidade de intubação e ventilação mecânica invasiva.

\section{Métodos}

Foi realizada uma busca nas bases de dados MEDLINE (acesso, via PubMed) e LILACS (acesso, via BIREME) por coortes que demonstrassem que a síndrome de fragilidade aumenta o risco de intubação e ventilação mecânica invasiva. Com o intuito de tornar a busca mais sensível, além do termo síndrome de fragilidade, foram incorporados e considerados também os marcadores clínicos da síndrome que podem apresentar associação à insuficiência respiratória, reconhecidos na literatura como sarcopenia, delírio, desnutrição. ${ }^{5}$ No MEDLINE, a busca foi realizada através de estratégia de busca específica, incluindo os termos de busca (Medical Subject Headings - MeSH) e respectivos sinônimos. Além disso, foi checada a lista de referências dos estudos incluídos. Esta estratégia de busca é exibida na Tabela 1. Já no LILACS, a busca foi feita através do termo "Frail Elderly" em todas as bases de dados indexadas. Relatos de caso, revisões narrativas e estudos de aplicação de inquéritos foram excluídos. Em ambas as bases de dados não houve restrição a idiomas e o processo de seleção dos estudos foi realizado por dois autores de for- 
ma independente. Eventuais diferenças foram resolvidas por consenso ou com a entrada de um terceiro membro da equipe de revisão, quando necessário. Até então, os dados não foram incluídos na revisão.

\section{Tabela 1}

Estratégia de busca utilizada no MEDLINE (acesso, via PubMed)

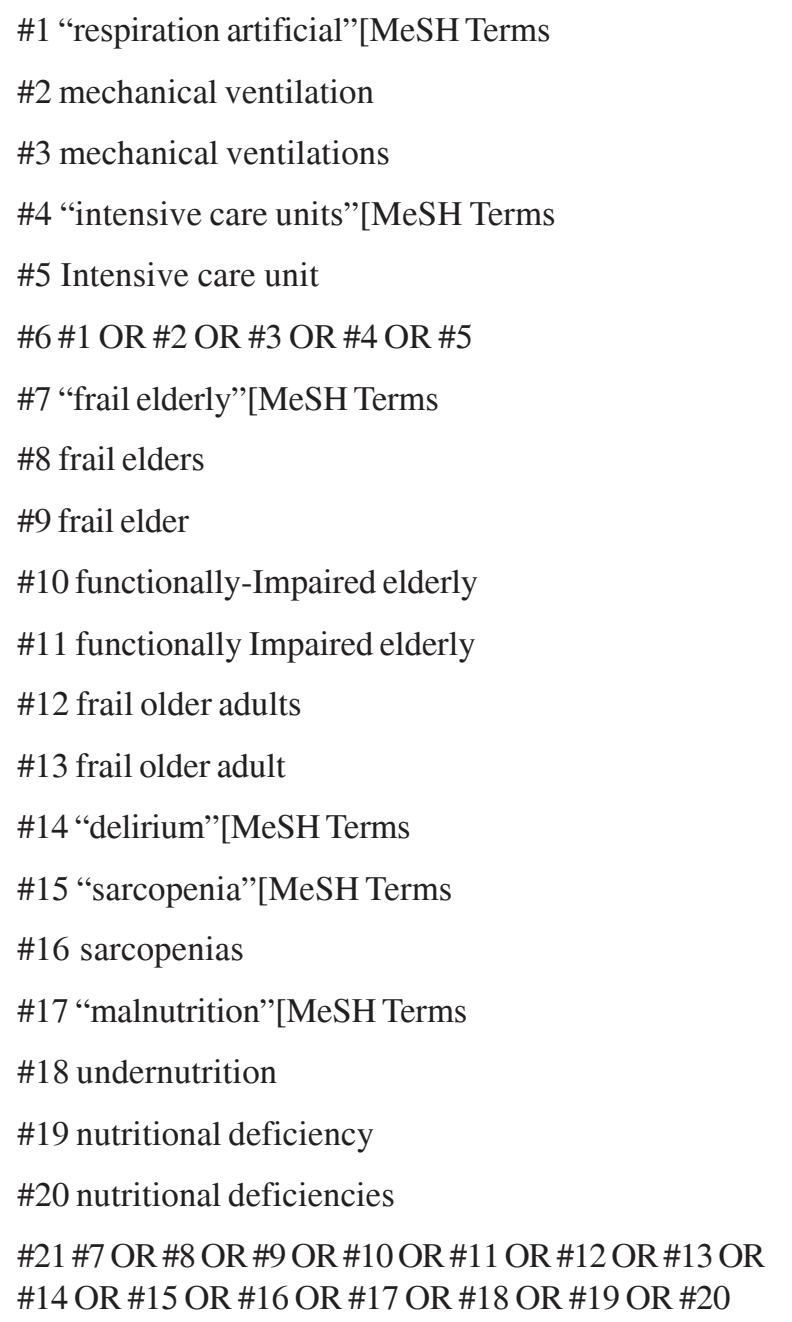

Os seguintes limites foram ativados para esta busca: "Aged: 65+ years, 80 and over: $80+$ years" e "published in the last 10 years".

\section{Resultados e comentários}

Nenhum estudo preencheu os critérios de inclusão desta revisão. Apenas um estudo foi considerado, potencialmente relevante para inclusão por avaliar um desfecho relacionado a um dos marcadores da síndrome de fragilidade citados acima. Seneff et al. avaliaram o Acute Physiology and Chronic Health
Evaluation III (APACHE III) com base em dados de 17.440 internações em UTI. Nesta população, a duração de tempo sob ventilação mecânica para pacientes que desenvolveram insuficiência respiratória esteve relacionada, principalmente, ao diagnóstico principal, e não à doença pulmonar subjacente. Desnutrição não foi preditivo, por si só, de quantidade de dias sob ventilação mecânica. ${ }^{15}$

Nós procuramos definir critérios bem sólidos para mapear os resultados em definições e condições da síndrome de fragilidade, não de envelhecimento. Embora a idade avançada claramente aumente o risco de desfechos negativos em UTI e haja uma estreita relação entre a síndrome de fragilidade e o envelhecimento, isso não significa que todos os idosos em estado crítico tenham um mau prognóstico. Diversos estudos, utilizando análise multivariada, têm demonstrado que a idade, por si só, não é um preditor independente de mortalidade. ${ }^{16-20}$ Secundariamente, seriam considerados estudos que avaliaram tal desfecho por marcadores clínicos da síndrome de fragilidade, na ausência de coortes sobre a essa alteração clínica. Como dito anteriormente, alguns marcadores clínicos da síndrome de fragilidade apresentam associação à insuficiência respiratória aguda, principalmente em idosos. ${ }^{5}$ Assim, nós buscamos informações que permitissem afirmar que há uma associação causal entre a síndrome de fragilidade e ventilação mecânica. No entanto, alguns comentários sobre possíveis motivos para ausência de evidências nessa área são pertinentes. Perspectivas distintas são encontradas na literatura para o entendimento do conceito da síndrome de fragilidade e tais modelos podem representá-lo por trajetórias diferentes ou caminhos para desfechos adversos, como incapacidade ou até mesmo morte ${ }^{1-21}$, apesar de, atualmente, o fenótipo de Linda Fried. ser o mais aceito para definir se um indivíduo é ou não frágil. ${ }^{22}$ Além disso, nesse fenótipo, do total de 5 critérios, pelo menos 3 devem estar presentes. ${ }^{3,5}$ Ou seja, estudos que porventura avaliem tais marcadores de forma isolada para desfechos de ventilação mecânica, em indivíduos que, apesar de apresentarem o indicador clínico da síndrome de fragilidade não possuam a síndrome diagnosticada, não nos permitem afirmar que há uma associação entre a síndrome de fragilidade e a ventilação mecânica. Além disso, a falta de evidências pode esbarrar, ainda, no conceito da limitação de idade para internações em UTIs e da relação custo/ benefício. ${ }^{23,24}$ Conforme citado anteriormente, diversos estudos concluíram que a idade não é um 
preditor de mortalidade. Mas, há argumentação quanto ao racionamento de recursos para a população ido$\mathrm{sa}^{24}$, pois, embora o conceito da síndrome de fragilidade se diferencie do conceito de envelhecimento, principalmente, por poder ser prevenida ${ }^{5}$, há uma estreita associação entre elas.

De forma contraditória, na literatura, há informações de que marcadores da síndrome aumentam a probabilidade dos pacientes, principalmente idosos, evoluírem a uma situação de insuficiência respiratória. ${ }^{5}$ Rooij et al. relatam que, na UTI, o delírio é preditivo de um aumento triplo nas taxas de reintubação e aumento médio de 10 dias internação. ${ }^{25}$ Alguns, no entanto, avaliam tais condições como preditivos de mortalidade, mas não como fatores de risco para ventilação mecânica. ${ }^{26}$

\section{Conclusões}

De uma forma geral, a síndrome de fragilidade é aceita como um estado de alta vulnerabilidade para resultados adversos da saúde, incluindo incapacidade, dependência, quedas, necessidade de cuidados em longo prazo, internação hospitalar e mortalidade. Conclusões de estudos anteriores indicam que a síndrome de fragilidade é uma entidade distinta, reconhecida pelos clínicos como uma síndrome de múltiplas manifestações possíveis e nenhuma manifestação única, por si só, é suficiente ou essencial na sua apresenta- ção. Muitos desses fatores estão interagidos e podem constituir, teoricamente, um ciclo da síndrome de fragilidade associado ao declínio da capacidade energética e de reserva. Algumas características da síndrome de fragilidade podem promover alterações de consciência e déficit muscular importante, o que aumentaria a probabilidade de evolução a uma situação de insuficiência respiratória, tratada, muitas vezes, com ventilação mecânica invasiva no âmbito da terapia intensiva. É provável que haja uma associação entre a síndrome de fragilidade e risco de morte, passando ou não pela ventilação mecânica invasiva. No entanto, estabelecer uma relação existente entre a síndrome de fragilidade e a ventilação mecânica invasiva se faz necessário, pois, por ser uma síndrome, ela pode ser prevenida e, dessa forma, seria possível uma redução da mortalidade ligada à essa alteração clínica. Com o reconhecimento clínico do estado de fragilidade, programas de reabilitação validados são capazes de postergar ou reduzir as suas graves consequiências, como o declínio funcional e a sarcopenia. Apesar de em nosso estudo não terem sido encontradas evidências de que a síndrome de fragilidade possa ser um fator de risco para ventilação mecânica invasiva, há indícios de uma provável relação entre ambas e uma urgente necessidade de realização de estudos que avaliem se marcadores clínicos da síndrome de fragilidade são fatores de risco para ventilação mecânica invasiva existe, com o intuito de responder a essa questão.

\begin{abstract}
Some clinical markers of frailty, such as malnutrition, sarcopenia and delirium are predictors of respiratory failure in the elderly. Thus, these risk factors can lead to patients considered vulnerable to greater susceptibility to intubation and mechanical ventilation. Objective: To identify evidence of a causal association between frailty and invasive mechanical ventilation. Methods: MEDLINE search for cohorts showing that the syndrome of frailty increases the risk of intubation and mechanical ventilation. Besides the term frailty, were incorporated and considered also markers of clinical syndrome that may have association with respiratory failure, found in the literature as sarcopenia, delirium, malnutrition. The search was performed using specific search strategy, including search terms (Medical Subject Headings - MeSH) and their synonyms. Furthermore, it was carry out additional search the reference list of included studies. Results: No study met the inclusion criteria of this review. Conclusions: In our study, we found no evidence that the weakness may be a risk factor for invasive mechanical ventilation. There are indications however, that delirium can increase the time under mechanical ventilation. Nevertheless, there is considerable lack of information in this area and a lot of uncertainty, perhaps because there is still no established consensus on the syndrome. There is an expressed need for studies to assess whether clinical markers of frailty are risk factors for invasive mechanical ventilation.
\end{abstract}

Key words: Frail Elderly. Aging. Intensive Care. Respiration, Artificial. 
Conflitos de interesse

Não há conflitos de interesse neste estudo.

\section{Referências}

1. Lang PO, Michel JP, Zekry D. Frailty syndrome: a transitional state in a dynamic. Process. Gerontology. 2009;55:539-49.

2. Fried LP, Watson J. Frailty and failure to thrice; in Ettinger WH, Halter JB, Ouslander JG (eds): Principles of Geriatric Medicine and Gerontology, ed 4. New York, McGraw-Hill Health Professions Divisions, 1998, PP 1387- 402.

3. Fried LP, Hadley EC, Walston JD et al. From bedside to bench: research agenda for frailty. SAGE KE 2005; 31:24.

4. Kim J, Davenport P, Sapienza C. Effect of expiratory muscle strength training on elderly cough function. Arch Gerontol Geriatr 2009; 48:361-6.

5. Sevransky JE, Haponik EF. Respiratory failure in elderly patients. Clin Geriatr Med 2003;19:205-24.

6. Sweeney RM, Barber V, Page $\mathrm{V}$ et al. A national survey of the management of delirium in UK intensive care units. Q J Med 2010;103:243-51.

7. Cadogan FL, Riekerk B, Vreeswijk R et al. Current awareness of delirium in the intensive care unit: a postal survey in the Netherlands. Neth J Med 2009;67:296-300.

8. Pun BT, Ely EW. The importance of diagnosing and managing ICU delirium. CHEST 2007;132:624-36.

9. Arend $\mathrm{E}$, Christensen M. Delirium in the intensive care unit: a review. Crit Care Nurse 2009;14:145-54.

10. Theou O, Jones GR, Ovenrend TJ et al. An exploration of the association between and muscle fatigue. Appl Physiol Nutr Metab 2008;33:651-65.

11. Fried LP, Tangen CM, Walston $\mathrm{J}$ et al. Frailty in older adults: evidence for a phenotype. J Gerontol 2001;56:M146-56.

12. Wu IC, Shiesh SC, Kuo PH et al. High oxidative stress is correlated with frailty in elderly chinese. American Geriatrics Society 2009;57:1666-71.
13. Marcell TJ. Sarcopenia: causes, consequences, and preventions. J Gerontol 2003; 58A:911-6.

14. Fried TR, Mor V. Frailty and hospitalization of long-term stay nursing home residents. J Am Geriatr Soc 1997;45:265-9.

15. Seneff MG, Zimmerman J, Knaus WA, et al. Predicting the duration of mechanical ventilation. CHEST 1996;110:469-79.

16. Hilmer SN, Perera V, Mitchell S, Dent J, Murnion BP, Bajorek B et al. The assessment of frailty in older people in acute care. Australas J Ageing. 2009;28:182-8.

17. Al Snih S, Graham JE, Ray LA et al. Frailty and incidence of activities of daily living disability among older mexican americans. J Rehabil Med 2009; 41: 892-7.

18. Rockwood K, Fox RA, Stolee P et al. Frailty in elderly people: an evolving concept. CMAJ 1994;150:489-95.

19. Kim J, Davenport P, Sapienza C. Effect of expiratory muscle strength training on elderly cough function. Arch Gerontol Geriatr 2009;48:361-6.

20. Fried LP, Watson J: Frailty and failure to thrice; in Ettinger WH, Halter JB, Ouslander JG (eds): Principles of Geriatric Medicine and Gerontology, ed 4. New York, McGraw-Hill Health Professions Divisions, 1998, PP 1387-402.

21. Cigolle CT, Ofstedal MB, Tian Z et al. Comparing models of frailty: the health and retirement study. J Am Geriatr Soc 2009;57:830-9.

22. Brasil. Ministério da Saúde. Secretaria de Atenção à Saúde. Departamento de Atenção Básica. Envelhecimento e saúde da pessoa idosa/ Ministério da Saúde, Secretaria de Atenção à Saúde, Departamento de Atenção Básica - Brasília: Ministério da Saúde, 2006.

23. Callahan D. Old age and new policy. JAMA 1989;261:905-6.

24. Levinsky NG. Age as a criterion for rationing health care. $N$ Engl J Med 1990;322:1813-6.

25. Rooij SE, Abu-Hanna A, Levi M et al. Factors that predict outcome of intensive care treatment in very elderly patients: a review. Crit Care. 2005;9:R307-R14.

26. Ely EW. Delirium as a predictor of mortality in mechanically ventilated patients in the intensive care unit. JAMA 2004 291:1753-62. 